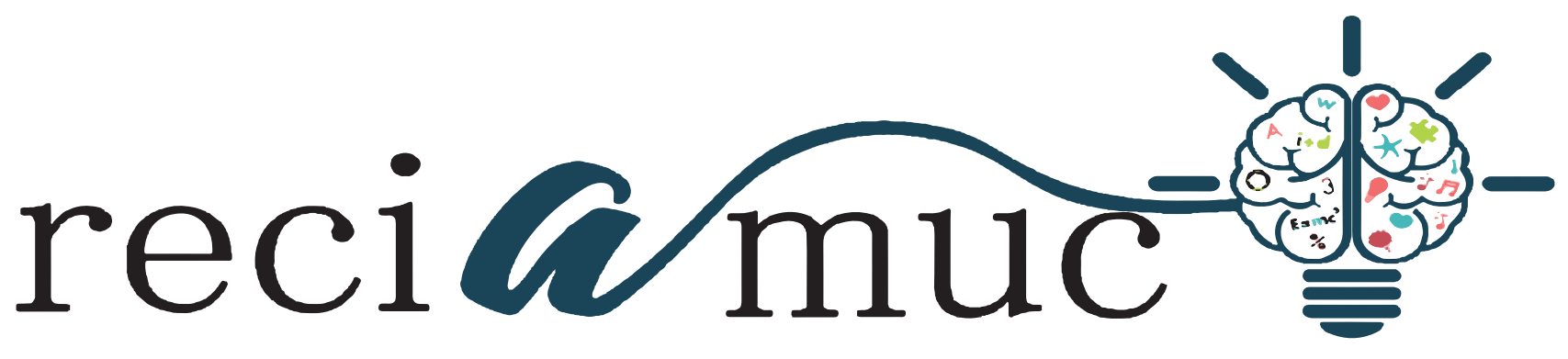

DOI: $10.26820 / \mathrm{reciamuc} / 4 .(4)$.diciembre.2020.64-73

URL: https://reciamuc.com/index.php/RECIAMUC/article/view/569

EDITORIAL: Saberes del Conocimiento

REVISTA: RECIAMUC

ISSN: 2588-0748

TIPO DE INVESTIGACIÓN: Artículo de Revisión

CÓDIGO UNESCO: 3102 Ingeniería Agrícola

PAGINAS: $64-73$

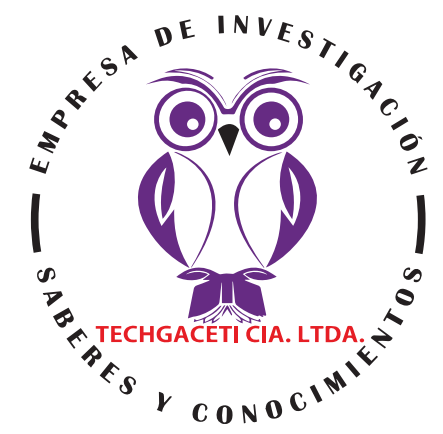

\title{
Presencia de Agroquímicos en muestra de Café en la Zona Sur de Manabí
}

Presence of Agrochemicals in coffee shows in the South Zone of Manabí

Presença de Agroquímicos em espectáculos de café na Zona Sul de Manabí Miguel Angel Osejos Merino'; Rocío Jaqueline Cano Andrade2; Sheila Johanna Cañarte Baque ${ }^{3}$

RECIBIDO: 23/09/2020 ACEPTADO: 17/10/2020 PUBLICADO: 24/12/2020

1. Doctor en Ciencias Ambientales; Diplomado en Autoevaluación y Acreditación Universitaria; Docente de la Universidad Estatal del Sur de Manabí; Jipijapa, Ecuador; miguel.osejos@unesum.edu.ec; https://orcid. org/0000-0001-7514-9510

2. Magister en Gerencia Educativa; Ingeniera Agrónoma; Docente de la Universidad Estatal del Sur de Manabí; Jipijapa, Ecuador; rocio.cano@unesum.edu.ec; https://orcid.org/0000-0002-1342-4705

3. Magister en Sistemas Integrados de Gestión; Diploma Superior en Auditoria de Gestión de la Calidad; Ingeniera Industrial; Docente de la Universidad Estatal del Sur de Manabí; Jipijapa, Ecuador; sheila. canarte@unesum. edu.ec; https://orcid.org/0000-0002-5181-8799

\section{CORRESPONDENCIA}

Miguel Angel Osejos Merino

miguel.osejos@unesum.edu.ec

Jipijapa, Ecuador;

(C) RECIAMUC; Editorial Saberes del Conocimiento, 2020 


\section{RESUMEN}

Nuestra investigación trata sobre los principales agroquímicos que se encuentran en el café en la Zona Sur de Manabí. La presente investigación tiene como propósito principal Identificar los residuos de agroquímicos en el café verde y las alternativas para el aseguramiento de la inocuidad del producto. La metodología empleada para su desarrollo se basó en la observación previo a la determinación del lugar y la aplicación de la toma de muestras, tabulación e interpretación de resultados. Cabe indicar que se efectuó la selección y tratamiento de las muestras de café en los meses de octubre y noviembre del periodo 2017, así como también en los meses de agosto y septiembre del 2018, con esto se realizó el análisis de pesticidas organoclorados y organofosforados en el café verde en el mes de diciembre del periodo 2017 y en el mes de octubre del 2018 por laboratorio GRUNTEC. Los resultados obtenidos fueron que los análisis de laboratorios realizados en los periodos 2017 y 2018 establecen que los productores de la zona sur de Manabí de los cantones Jipijapa, Pajan y 24 de Mayo no aplican productos químicos en el manejo de sus plantaciones de café.

Palabras clave: Agroquímicos, Calidad de Café, Zona Sur de Manabí.

\section{ABSTRACT}

Our research deals with the main agrochemicals found in coffee in the South Zone of Manabí. The main purpose of this research is to identify the agrochemical residues in green coffee and the alternatives to ensure the safety of the product. The methodology used for its development was based on observation prior to the determination of the place and the application of sampling, tabulation and interpretation of results. It should be noted that the selection and treatment of the coffee samples was carried out in the months of October and November of the 2017 period, as well as in the months of August and September 2018, with this the analysis of organochlorine and organophosphate pesticides was carried out in green coffee in the month of December of the period 2017 and in the month of October 2018 by GRUNTEC laboratory. The results obtained were that the laboratory analyzes carried out in the periods 2017 and 2018 establish that the producers of the southern zone of Manabí in the cantons Jipijapa, Pajan and 24 de Mayo do not apply chemical products in the management of their coffee plantations.

Keywords: Agrochemicals, coffee quality, South Zone of Manabí.

\section{RESUMO}

A nossa investigação trata dos principais agroquímicos encontrados no café na Zona Sul de Manabí. O principal objectivo desta investigação é identificar os resíduos agroquímicos no café verde e as alternativas para garantir a segurança do produto. A metodologia utilizada para o seu desenvolvimento foi baseada na observação prévia à determinação do local e à aplicação de amostragem, tabulação e interpretação dos resultados. É de notar que a selecção e tratamento das amostras de café foi realizada nos meses de Outubro e Novembro do período 2017, bem como nos meses de Agosto e Setembro de 2018, com isto a análise dos pesticidas organoclorados e organofosforados foi realizada no café verde no mês de Dezembro do período 2017 e no mês de Outubro de 2018 pelo laboratório GRUNTEC. Os resultados obtidos foram que as análises laboratoriais realizadas nos períodos 2017 e 2018 estabelecem que os produtores da zona sul de Manabí nos cantões Jipijapa, Pajan e 24 de Mayo não aplicam produtos químicos na gestão das suas plantações de café.

Palavras-chave: Agroquímicos, qualidade do café, Zona Sul de Manabí. 


\section{Introducción}

La caficultura ecuatoriana y particularmente de la zona Sur de la provincia de Manabí en los últimos años, se encuentra en una situación crítica debido a la baja productividad y deficiente calidad del grano de exportación, debido a la ubicación del cultivo en zonas marginales, la prevalencia de cafetales viejos e improductivos y la no adopción masiva de las tecnologías apropiadas de producción y post-cosecha.

El sector cafetalero ecuatoriano, tiene varias fortalezas como la amplia diversidad de agro ecosistemas, muchos de ellos con aptitudes para producir café finos tipo "gourmet", la capacidad instalada de la industria del café con una alta demanda de materia prima, la disponibilidad de tecnología apropiada, la consolidación de gremios de productores, una elevada población trabajadora vinculada a la caficultura y el apoyo de varias instituciones nacionales y de la cooperación internacional a las organizaciones de caficultores en el proceso de construcción de alternativas sustentables.

La presente investigación se fundamentó en la urgencia de Identificar los residuos de agroquímicos en el café verde y las alternativas para el aseguramiento de la inocuidad del producto en la Zona Sur de la Provincia de Manabí, con las prácticas en la post cosecha más apropiadas para asegurar una adecuada calidad física y organoléptica del grano y la bebida, asegura la inocuidad del producto, esto significa que el producto debe garantizar la calidad e inocuidad al consumidor final.

La calidad del café se define por la evaluación de sus características físicas y organolépticas que son importantes para la comercialización del grano (Puerta, 1999; Fischersworring y Robkamp, 2001).

Estas pruebas de la calidad del café son efectuadas por profesionales expertos denominados catadores quienes, a través de su experiencia y entrenamiento, determinan las características que presenta tanto el grano como la bebida (Haarer, 1982).

La calidad del café se determina evaluando una serie de características que varían con las necesidades y gustos de los consumidores (Menchú, 1967).

Los productores cafetaleros y la Universidad Estatal del Sur de Manabí, consideran que la iniciativa en implementar esta investigación que derivo de uno de los objetivos específicos del proyecto de Investigación de "Mejoramiento de la calidad e inocuidad del café", beneficia directamente al proceso de reactivación de la caficultura; sus resultados permitieron saber la calidad de café que producen las fincas de los productores de la zona sur de Manabí, con lo que garantizan ofrecer al mercado nacional e internacional el café de buena calidad.

\section{Marco teórico}

\subsection{Agroquímicos más comunes en mues- tra de Café.}

El DDT.- (diclorodifeniltricloroetano) Es un plaguicida usado extensamente en el pasado para controlar insectos en cosechas agrícolas e insectos portadores de enfermedades tales como la malaria y el tifus. Actualmente se usa solamente en unos pocos países para controlar la malaria. (Departamento de salud y servicios humanos de los EE.UU.,2002).

El DDT técnico es de una composición variable y puede consistir en 11 o más compuestos, principalmente el isómero pp'(hasta un 70\%) y el isómero op'-(15 al 30\%). El pp' DDT es un sólido cristalino blanco, la mezcla técnica es un sólido ceroso de color blanco, o crema o un polvo amorfo. Según el grado de toxicidad la OMS lo clasifica como clase II, moderadamente peligroso. (Departamento de salud y servicios humanos de los EE.UU.,2002).

El 2,4,5-T.- Los herbicidas clorofenoxi, como 2,4-D, 2,4,5-T, MCPA y silvex, son reguladores del crecimiento. Actúan como auxinas 
sintéticas u hormonas de crecimiento, alterando el metabolismo de la planta y posterior crecimiento. La selectividad de estos compuestos es el resultado de las diferencias inherentes entre las respuestas de los sistemas enzimáticos de cultivos y de malezas (Simoniello, 2011).

Debido a la prisa por satisfacer la demanda de Agente Naranja por parte del ejército estadounidense, el producto se contaminó durante el proceso de producción con la dioxina TCDD (la 2,3,7,8-tetraclorodibenzodioxina). La TCDD es un inevitable e indeseable subproducto de la fabricación del 2,4,5-T. En uso comercial, el TCDD estaba presente en el herbicida en concentraciones muy inferiores, 0,05 partes por millón (ppm), mientras que en los lotes de herbicida enviados a Vietnam llegaba a alcanzar las 50 ppm. Como consecuencia la contaminación por dioxinas debida al Agente Naranja fue 1.000 veces superior que la que ocasionaban los herbicidas agrícolas. Se estima que la TCDD es la más tóxica de las dioxinas, una familia de compuestos químicos que ha sido descrita como el grupo de las sustancias más tóxicas para los humanos que se conocen (Breccia \& Santiago, 2018).

El ácido 2,4,5-triclorofenoxiacético se utiliza en la explotación forestal y en agricultura como herbicida sistémico (para la erradicación de malezas, entre otros).

El 2,4,5-T en las plantas como efecto característico actúa como efectivo defoliante. Las plantas (y en particular las dicotiledóneas) absorben el 2,4,5-T a través de sus hojas y metabolizan la sustancia. Se inhiben numerosos procesos metabólicos. El efecto directo se presenta en algunas ocasiones como problemas con la permeabilidad pasiva, pero también se refleja en un incremento de la formación de oxígeno y mayor crecimiento. El 2,4,5-T también actúa como desacoplador de la cadena respiratoria. (Centro de Ciencias Ecológicas Instituto Indio de Ciencia Bangalore 560 012).
En el suelo, el ácido 2,4,5-triclorofenoxiacético se degrada por acción microbiana o se evapora a la atmósfera. La mayor parte del herbicida permanece en el horizonte superior del suelo (hasta profundidades de $10 \mathrm{~cm}$ ), debido a su moderada movilidad en este medio. Las plantas extraen considerables cantidades de 2,4,5-T del suelo. A más de $500^{\circ} \mathrm{C}$ la sustancia se destruye por acción de la temperatura y se forma TCDD. En un ambiente ácido permanece estable. En el suelo, la degradación se debe principalmente a los microorganismos; la degradación a CO2 se acelera por efecto de las sustancias húmicas y ácidos fúlvicos. El ácido 2,4,5-triclorofenoxiacético se forma en sedimentos anaeróbicos y durante la fotólisis en presencia de sustancias húmicas. El 2,4,5-T se transforma en clorofenoles, polifenoles, quinonas y productos similares a los ácidos húmicos. (Centro de Ciencias Ecológicas Instituto Indio de Ciencia Bangalore 560 012).

El 2,4-D.- El ácido 2,4- diclorofenoxiacético (CAS 94-75-7) fue descrito por primera vez en 1942 como un auxin sintético, es decir un tipo de hormona de las plantas. Esta substancia es usada como un herbicida sistémico y actúa como un inhibidor del crecimiento, como puede notarse en la curvatura de las hojas que provoca dicho compuesto. Las sales son absorbidas por las raíces mientras los ésteres son principalmente absorbidos por las hojas. Controla las malezas de hojas anchas (dicotiledóneas) mientras las monocotiledóneas como por ejemplo los cereales (incluyendo el maíz) y las plantas herbáceas (incluyendo la caña de azúcar y el bambú) en su mayoría no se ven afectadas por este ácido (oPM 2012). La tolerancia natural de las monocotiledóneas es de cierta forma limitada, ya que por ejemplo sí afecta a las plantas de maíz en las etapas más tardías de vida. (Pesticides Action Network, 2014).

El herbicida 2,4-D ha sido ampliamente utilizado para el control de malezas en la agricultura, la silvicultura y los entornos urbanos

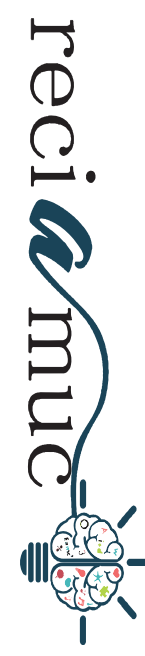


y residenciales. Las exposiciones ocupacionales a 2,4-D pueden ocurrir durante la fabricación y aplicación, y la población en general puede estar expuestos a través de los alimentos, el agua, el polvo, o la aplicación residencial, y durante la pulverización. (Pesticides Action Network, 2014).

El herbicida 2,4-D fue clasificado como posible carcinógeno para los humanos (Grupo 2B), basado en evidencias insuficientes en seres humanos y en evidencia limitada en animales de experimentación. Los estudios epidemiológicos proporcionan una fuerte evidencia de que el 2,4-D induce estrés oxidativo, un mecanismo que puede operar en los seres humanos, y pruebas moderadas de que el 2,4-D provoca inmunosupresión, basados en estudios in vivo e in vitro. Sin embargo, los estudios epidemiológicos no encontraron un aumento importante o constante de riesgo de LNH u otros tipos de cáncer en relación con la exposición del herbicida 2,4-D. (Pesticides Action Network, 2014).

El Paraquat.- Es un herbicida bipiridílico que actúa por contacto, se presenta en forma líquida en concentraciones del 20\% para uso agrícola. Su nombre químico es el 1-1'-dimetil-4-4- bipiridilos, con el nombre comercial de Gramoxone. La intoxicación por paraquat tiene una tasa de mortalidad elevada. La intoxicación grave se caracteriza por la afectación de múltiples órganos, principalmente los pulmones, los riñones y el hígado. El pulmón es el órgano diana en la intoxicación por paraquat y la insuficiencia respiratoria con fibrosis pulmonar aguda es la causa más común de muerte. (Glenda, 2014).

Su uso es frecuente en zonas agrícolas. Se encuentra al alcance de los agricultores desde hace más de 40 años, es el segundo agroquímico más vendido en el mundo. Las propiedades herbicidas del paraquat fueron descubiertas en 1955 y el principio activo fue introducido en los mercados mundiales en 1962 con el nombre de marca
GRAMOXONE®, presentación en solución acuosa al 20 o al 40\%. Su acción herbicida se ejerce por idénticos mecanismos que su acción tóxica ya que bloquea los procesos de respiración celular. (Glenda, 2014).

La OMS en su Clasificación Recomendada de Plaguicidas según sus riesgos, clasifica el paraquat como "Moderadamente peligroso, clase II". La dosis letal mínima estimada para humanos es $10-15 \mathrm{ml}$ del producto concentrado, dosis letal media 110 - 150 $\mathrm{mg} / \mathrm{kg}$ por vía oral en ratas. (Glenda, 2014).

La inhalación de paraquat puede causar daño pulmonar y puede conducir a una enfermedad Ilamada pulmón de paraquat. El paraquat causa daño al cuerpo cuando entra en contacto con el revestimiento de la boca, el estómago o los intestinos. Una persona se puede enfermar si el paraquat entra en contacto con una herida de la piel. Este herbicida también puede causar daño a los riñones, el hígado y el esófago (el tubo que lleva la comida desde la boca hasta el estómago. La ingestión de paraquat puede causar rápidamente la muerte, la cual puede ocurrir a raíz de un orificio en el esófago o por una inflamación grave de la zona que rodea los grandes vasos sanguíneos y las vías respiratorias en la parte media del tórax. La exposición a largo plazo al paraquat puede causar cicatrización en los pulmones llamada fibrosis pulmonar. Esto dificulta la respiración. (Glenda, 2014).

El parathión.- Es un plaguicida organofosforado prohibido en todas sus formulaciones y usos por ser dañino para la salud humana; animal y el ambiente. Parathión es el nombre común de un insecticida organofosforado que se usó en el pasado en los Estados Unidos y que aún se usa en otros países para controlar insectos y ácaros que chupan o mascan las plantas en una extensa variedad de cosechas. (Agencia para Sustancias Tóxicas y el Registro de Enfermedades, 2002).

Es un potentísimo insecticida y acaricida extremadamente tóxico, con pobre poder 
residual. Fue originalmente desarrollado por IG Farben en los 1940. Es altísimamente tóxico para todos los organismos de vida, incluyendo humanos. En algunos países solo está restringido su uso, y hay propuestas para prohibirlo en todos sus usos; está estrechamente relacionado con el "metil paratión". (Agencia para Sustancias Tóxicas y el Registro de Enfermedades, 2002).

El parathión fue excluido de la lista de sustancias activas autorizadas para el uso en productos de protección de plantas en 1970 bajo la Ley para protección de plantas contra plagas y pestes, Prohibida la producción, uso y comercialización de todos los productos de protección de plantas que contengan Paratión de acuerdo a la lista anual adoptada de ingredientes activos prohibidos para el uso en productos de protección de plantas, bajo el Acta de Protección de Plantas desde 2003. (Agencia para Sustancias Tóxicas y el Registro de Enfermedades, 2002).

La producción y uso de parathión como insecticida en el pasado llevó a su liberación al aire, al agua y al suelo. El parathión en el aire es transformado rápidamente por la luz solar y ozono en un producto de degradación, paraoxón, una sustancia más tóxica que el parathión. En el agua, el parathión puede ser degradado por la luz solar y por microorganismos. En el agua, la reacción con otras sustancias químicas y con la luz solar produce paraoxón. En el suelo, el parathión puede ser degradado por reacciones químicas, la luz solar, o microorganismos. (Agencia para Sustancias Tóxicas y el Registro de Enfermedades, 2002).

El aldrín.- Es un plaguicida que ha sido muy ampliamente utilizado como insecticida, aunque ha dejado de usarse debido principalmente a su carácter persistente en el medio ambiente y a su tendencia a la bioacumulación en la cadena alimenticia por su alta solubilidad en grasas. El aldrín y el dieldrín son insecticidas y pesticidas organoclorados. Tienen características de liposolu- bilidad, bioacumulación y persistencia en el medio ambiente (Contaminantes Orgánicos Persistentes - COP), y además pueden ser transportados a grandes distancias por vía atmosférica. (Agencia para Sustancias Tóxicas y el Registro de Enfermedades, 2002).

\subsection{Calidad del café.}

La calidad se define como la aptitud de un producto para satisfacer la necesidad de un consumidor específico. (Comité Departamental de Cafeteros de Antioquia, 1991).

La calidad del café se define por la evaluación de sus características físicas y organolépticas que son importantes para la comercialización del grano (Puerta, 1999; Fischersworring y Robkamp, 2001). Estas pruebas son efectuadas por profesionales expertos denominados catadores quienes, a través de su experiencia y entrenamiento, determinan las características que presenta tanto el grano como la bebida (Haarer, 1982). La calidad del café se determina evaluando una serie de características que varían con las necesidades y gustos de los consumidores (Menchú, 1967).

Las dos especies de café más cultivadas en el mundo y de mayor importancia comercial son: Coffea arábica L. y Coffea Canephora P., que tienen marcadas diferencias genéticas, morfológicas, composición química y caracteres organolépticos. Los cafés arábigos producen una bebida suave, de aroma agradable, buena acidez y mediano cuerpo. El café robusto es de sabor amargo, aroma y acidez bajos, pero de buen cuerpo (Hein et al., 1992; Puerta, 2000). El café arábigo posee un color verde azulado, con un contenido de cafeína entre 0.9 a 1.2 por ciento.

Los factores que determinan la calidad del café son: 1) la composición química del grano (condicionada por la composición genética de la especie y la variedad); 2) las técnicas de cultivo; 3) los factores climáticos; 4) las prácticas culturales; 5) los métodos de cosecha; 6) el tipo de beneficio; 7) el secado; 8) el almacenamiento; y, 9) el gusto 
del consumidor (Coste, 1969; Hein et al., 1992).

Los denominados sistemas de control de calidad son un mecanismo que establecen y garantizan los estándares de calidad producidos en los países exportadores de café. Para discutir aspectos de la calidad del café arábigo es necesario dar una descripción del producto ideal buscado por los compradores (Wallis, 1967).

\section{Metodología}

La presente investigación se realizó en el sector cafetalero de la zona sur de Manabí, en los cantones de Jipijapa, Pajan y 24 de Mayo. Las Condiciones climáticas de la zona sur de Manabí son: Temperatura promedio de $18-28^{\circ} \mathrm{C}$, precipitación media anual de 200 a 300 $\mathrm{mm}$. por año, la topografía es Irregular, la zona de vida es bosque seco tropical, la altitud es de 250 msnm., la humedad Relativa de 35-45 \%, la textura es franco arcilloso-arcilloso y el Ph Neutro.

El tipo de investigación es de campo y de laboratorio. La presente investigación fue desarrollada mediante el método de observación previo a la determinación del lugar y la aplicación de la toma de muestras, tabulación e interpretación de resultados. Cabe indicar que se realizó la contratación del proveedor de muestras de café verde natural y pergamino seco en 120 productores de los cantones Jipijapa, Pajan y 24 de Mayo, luego se efectuó la selección y tratamiento de las muestras de café en los meses de octubre y noviembre del periodo 2017, así como también en los meses de agosto y septiembre del 2018, con esto se realizó el análisis de pesticidas organoclorados y organofosforados en el café verde en el mes de diciembre del periodo 2017 y en el mes de octubre del 2018 por laboratorio GRUNTEC Servicios Ambientales, en la ciudad de Quito-Ecuador. El número de muestra de café verde fue de 55 muestras en el año 2017 y 50 en el año 2018.

\section{Resultados}

Tabla 1. Reporte de Análisis de Pesticidas Organoclorados y Organofosforados en el Café verde realizado en diciembre del 2017 por Laboratorio GRUNTEC.

\begin{tabular}{|c|c|c|c|l|}
\hline $\begin{array}{c}\text { Nombre del } \\
\text { producto }\end{array}$ & Grupos & Rango mg./Kg & $\begin{array}{l}\text { Fecha de } \\
\text { muestreo }\end{array}$ & $\begin{array}{l}\text { Método } \\
\text { Adaptado de } \\
\text { referencia } \\
\text { Método interno }\end{array}$ \\
\hline $2,4,5, T$ & Organoclorado & $<0,02$ & Diciembre 2017 & $\begin{array}{l}\text { P-14.098, GC- } \\
\text { MS }\end{array}$ \\
\hline Paraquat & Organofosforado & $<0,02$ & Diciembre 2017 & $\begin{array}{l}\text { LA-LCMS- } \\
\text { 046,LC-MS/MS }\end{array}$ \\
\hline $2,4, \mathrm{D}$ & Organoclorado & $<0,01$ & Diciembre 2017 & $\begin{array}{l}\text { P-14.098,GC- } \\
\text { MS }\end{array}$ \\
\hline Parathion & Organofosforado & $<0,01$ & Diciembre 2017 & GC - FPD \\
\hline
\end{tabular}

Fuente: Investigación de campo.

Elaboración Propia. 
Gráfico 1. Reporte de Análisis de Pesticidas Organoclorados y Organofosforados en el Café verde realizado en diciembre del 2017 por Laboratorio GRUNTEC.

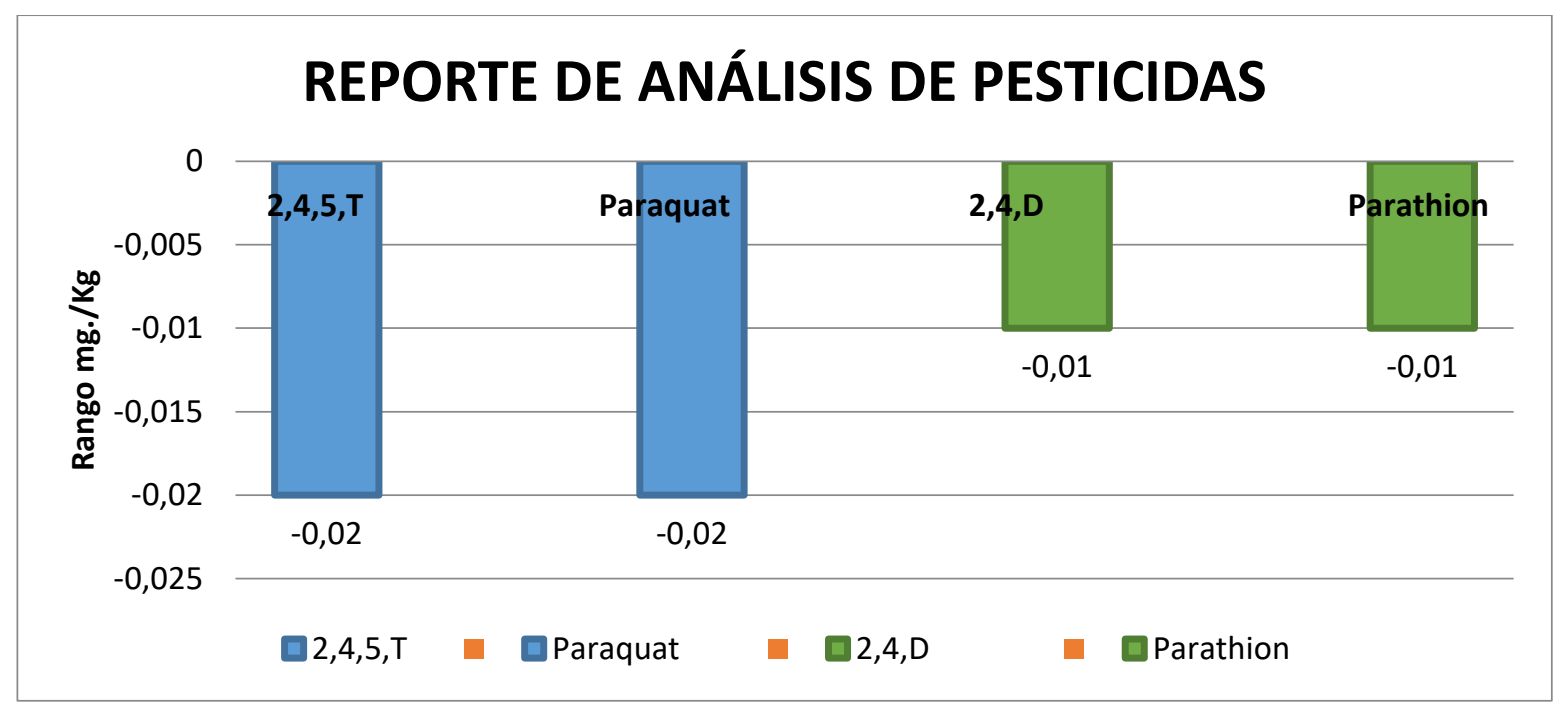

Fuente: Investigación de campo.

Elaboración Propia.

Analizado e interpretado los resultados de la tabla y grafico $N^{\circ} 1$ se puede apreciar que en el mes de diciembre del periodo 2017 en los análisis de las muestras de café verde se demostró que los pesticidas 2,4,5T, Paraquat, 2,4,D y Paration fueron no significativos lo que determinó que los valores obtenidos de acuerdo a los rangos estadísticos no superan los parámetros permisibles.

Tabla 2. Reporte de Análisis de Pesticidas Organoclorados y Organofosforados en el Café verde realizado en octubre del 2018 por laboratorio GRUNTEC.

\begin{tabular}{|c|c|c|c|c|}
\hline $\begin{array}{c}\text { Nombre del } \\
\text { producto }\end{array}$ & Grupos & Rango mg./Kg & $\begin{array}{l}\text { Fecha de } \\
\text { muestreo } \\
23 / 10 / 2018\end{array}$ & $\begin{array}{l}\text { Método } \\
\text { Adaptado de } \\
\text { referencia } \\
\text { Método interno }\end{array}$ \\
\hline Paraquat & Organofosforado & $<0,02$ & Octubre 2018 & LC-MS/MS \\
\hline $2,4,5, T$ & Organoclorado & $<0,02$ & Octubre 2018 & LC-MS/MS \\
\hline $2,4, \mathrm{D}$ & Organoclorado & $<0,01$ & Octubre 2018 & LC-MS/MS \\
\hline Parathion & Organofosforado & $<0,01$ & Octubre 2018 & $\begin{array}{l}\text { EPA 8270D/MM- } \\
\text { AG/S/BEG-27 }\end{array}$ \\
\hline
\end{tabular}

Fuente: Investigación de campo.

Elaboración Propia. 
Gráfico 2. Reporte de Análisis de Pesticidas Organoclorados y Organofosforados en el Café verde realizado en octubre del 2018 por laboratorio GRUNTEC.

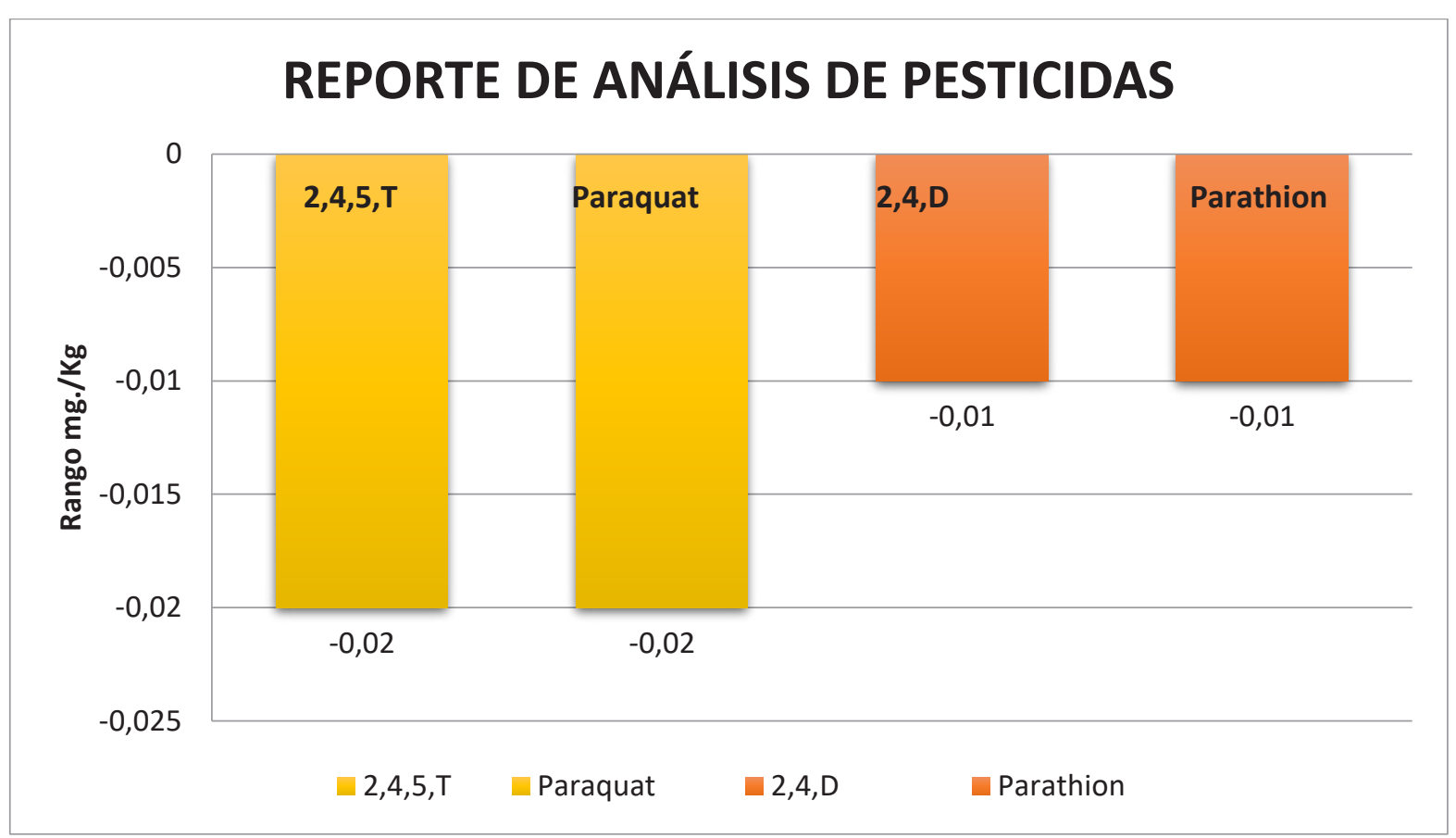

Fuente: Investigación de campo.

Elaboración Propia.

Analizado e interpretado los resultados de la tabla y grafico $\mathrm{N}^{\circ} 2$ se puede apreciar que en el mes de octubre del periodo 2018 en los análisis de las muestras de café verde se demostró que los pesticidas 2,4,5.T, Paraquat, 2,4,D y Paration fueron no significativos lo que determinó que los valores obtenidos de acuerdo a los rangos estadísticos no superan los parámetros permisibles.

\section{Conclusiones}

- Se comprobó en el mes de diciembre del periodo 2017 y en el mes de octubre del periodo 2018, con los análisis de las muestras de café verde que los pesticidas 2,4,5, T, Paraquat, 2,4,D y Paration fueron no significativos, con esto se determinó que los valores obtenidos de acuerdo a los rangos estadísticos no superan los parámetros permisibles.

- Los resultados de los análisis de laboratorios GRUNTEC del periodo 2017 y 2018 establecen que los productores de la zona sur de Manabí de los cantones Jipijapa, Pajan y 24 de Mayo no aplican productos químicos en el manejo de sus plantaciones de café.

\section{Agradecimientos}

Agradecemos a las autoridades de la Universidad Estatal del Sur de Manabí (UNESUM), por el apoyo en esta investigación.

\section{Bibliografía}

Agencia para Sustancias Tóxicas y el Registro de Enfermedades, (2002). Reseña Toxicológica de el Aldrín y el Dieldrín. Atlanta: GA: Departamento de Salud y Servicios Humanos de EE. UU., Servicio de Salud Pública.

Breccia, M., \& Santiago, E. (2018). Residuos de Plaguicidas en Alimentos.

Centro de Ciencias Ecológicas Instituto Indio de Ciencia Bangalore 560 012, I. (s.f.). Sistema de información ambiental.

Comité Departamental de Cafeteros de Antioquia. 1991. El Beneficio del Café. Calidad del café. Colombia. 209 p. 
Coste, R. 1969. El Café. Ed. Blume. Barcelona, España. pp. 185-199.

Departamento de Salud y Servicios Humanos de los EE.UU., S. d. (2002). Resumen de Salud Pública DDT, DDE y DDD .

Fischersworring, B., y Robkamp, R. 2001. Guía para la Caficultura Ecológica. GTZ. Popayán, Colombia. pp. 133-135.

Glenda, L. (2014). Intoxicación por Paraquat. Medicina Legal de Costa Rica On-line version ISSN 2215-5287Print version ISSN 1409-0015.

Haarer, E. 1982. Producción Moderna del Café. México. pp. 394-397.

Hein Jan Van Hilter, Marc Van de Steene, Abba Bayer. 1992. Café: Guía del Exportador. Centro de Comercio Internacional UNCTAD/GATT. Ginebra, Suiza. pp. 328-338.

Menchú, F. 1967. La determinación de la calidad del café. Parte I. En: Revista Agricultura de las Américas $N^{\circ}$ 5. Guatemala. pp. 18-21.
Menchú, F. 1967. La determinación de la calidad del café. Parte III. En: Revista Agricultura de las Américas № 8. Guatemala. pp. 36-37.

Pesticides Action Network, P. (2014). Riesgos del Herbicida 2,4 - D.

Puerta, Q., G.I. 1999. Influencia del proceso de beneficio en la calidad del café. Chinchiná, Caldas, Colombia. Federación Nacional de Cafeteros de Colombia. CENICAFE. 50 (1): 78-88.

Puerta, Q., G.I. 2000. Calidad de taza de algunas mezclas de variedades de café de la especie Coffea arábica L. Chinchiná, Caldas, Colombia. Federación Nacional de Cafeteros de Colombia. CENICAFE. 51 (1): 5-19.

Simoniello, F. (2011). Exposición ocupacional a los Agroquímicos. Evaluación del Daño Genético y su relación con procesos de Estrés Oxidativo. Argentina.

Wallis, J. A. N. 1967. Café: La calidad del café arábigo en Kenia. Volumen 8 (1 y 2). Perú. 33 P.

\section{CITAR ESTE ARTICULO:}

Osejos Merino, M. A., Cano Andrade, R. J., \& Cañarte Baque, S. J. (2020). Presencia de Agroquímicos en muestra de Café en la Zona Sur de Manabí. RECIAMUC, 4(4 (esp), 64-73. https://doi.org/10.26820/reciamuc/4.(4).diciembre.2020.64-73 